\title{
INVESTIGATING THE COHERENT NORMALIZATION METHOD FOR A BONE GADOLINIUM X-RAY FLUORESCENCE MEASUREMENT SYSTEM: A MONTE CARLO STUDY
}

By

\author{
Zaid Keldani \\ BEng, UOIT, 2015 \\ Oshawa, Ontario, Canada
}

\author{
A thesis \\ presented to Ryerson University \\ in partial fulfillment of the \\ requirements for the degree of \\ Master of Science \\ in the program of \\ Biomedical Physics
}

Toronto, Ontario, Canada, 2017

(C) Zaid Keldani 2017 


\section{AUTHOR'S DECLARATION FOR ELECTRONIC SUBMISSION OF A THESIS}

I hereby declare that I am the sole author of this thesis. This is a true copy of the thesis, including any required final revisions, as accepted by my examiners.

I authorize Ryerson University to lend this thesis to other institutions or individuals for the purpose of scholarly research.

I further authorize Ryerson University to reproduce this thesis by photocopying or by other means, in total or in part, at the request of other institutions or individuals for the purpose of scholarly research.

I understand that my thesis may be made electronically available to the public. 


\author{
Abstract \\ Investigating the Coherent Normalization Method for a Bone Gadolinium X-ray \\ Fluorescence Measurement System: A Monte Carlo Study \\ Zaid Keldani \\ Master of Science, Biomedical Physics \\ Ryerson University, 2017
}

Elevated gadolinium levels in patients with healthy renal function exposed to gadoliniumbased contrast agents (GBCA) have been confirmed by studies in the literature. Though the potential long-term effects of the retained gadolinium are still unknown, symptoms such as bone and joint pain have been reported in some subjects. As detecting the presence of gadolinium in vivo is required to diagnose toxicity related medical conditions, a ${ }^{109} \mathrm{Cd}$ based X-ray fluorescence (XRF) bone gadolinium measurement system has been previously developed. The current method is dependent on geometrical factors and other interpatient factors, such as the size and shape of the bone and the tissue thickness overlying the measurement site, which reduces the robustness of the measurement system and causes the need for correction factors.

It was previously shown that the successful use of the coherent normalization procedure can eliminate the need for such corrections and is conditional on four criteria that must be met. When detecting gadolinium two of the four criteria are not satisfied which makes further investigation of the method required. 
This work investigates the feasibility of the coherent normalization method to correct for the effect of varying overlying tissue thickness of an adult population through Monte Carlo simulations.

The coherent normalization method was studied as a function of overlying tissue thickness (OTT) to represent varying body types. The average coherent ratio (Gd K X-ray counts/Coherent counts) was found to be $0.717 \pm 0.025$ and the normalization resulted in a line with a slope of $-0.0053 \pm 0.0040$ which is insignificant at the $95 \%$ confidence level ( $\mathrm{p}=0.43$ ) suggesting the validity of the method. Additionally, this thesis provides a theoretical explanation of the feasibility of the method regardless of not fulfilling all four criteria, through introducing the Secondary Fluence Fluorescence Factor (SFFF) and separating fluence components, primary and secondary, contributing to the fluorescence of gadolinium. 


\title{
ACKNOWLEDGMENTS
}

\author{
"Commit to the LORD whatever you do, and he will establish your plans" \\ [Proverbs 16:3]
}

I owe my deepest gratitude to my supervisor, Dr. James Gräfe, for his guidance and constant support that made completing this thesis possible. Dr. Gräfe is a great supervisor, his door was always open for my questions. Working under his supervision has been a pleasure.

I am grateful to my supervisory committee; Dr. Ana Pejović-Milić, Dr. Darrick Heyd and Dr. Eric Da Silva for their valuable experience and input in this project. Many thanks to Dr. Pejović-Milić for lending me equipment and Dr. Da Silva for sharing his expertise in making bone phantoms.

To my parents, Randa and George, and brothers, Said and Mutaz, my words can never be enough to thank you for your love and support in every step of my life. I would also like to thank my uncle, Fr. Dr. Hanna Kildani, for his continuous encouragement.

I would like to acknowledge my colleagues Ms. Michelle Lord and Ms. Joanna Nguyen for their help that made completing this project possible. And to my fellow graduate students at the department of physics, thank you for filling this journey with many fun memories and great laughs.

Last but not least, many thanks to all faculty members and departmental staff for their warm smiles and endless support. 


\section{TABLE OF CONTENTS}

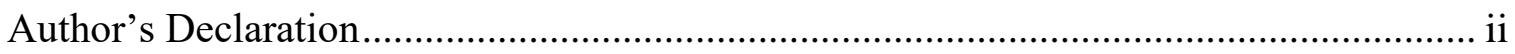

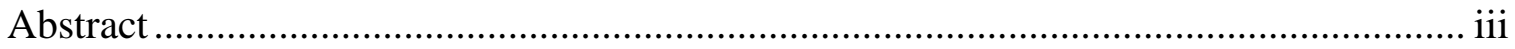

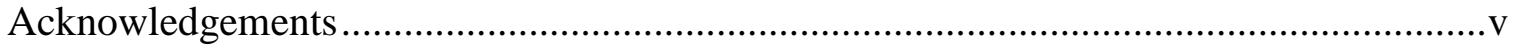

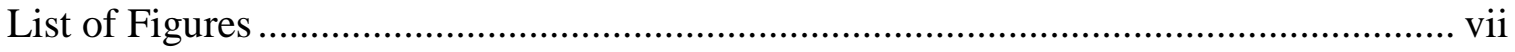

List of Symbols and Abbreviations....................................................................... viii

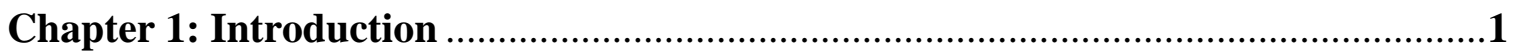

1.1 Gadolinium Toxicity ......................................................................................

1.2 X-ray Fluorescence Spectroscopic Technique ………......................................5

1.3 Coherent Normalization Method ...................................................................

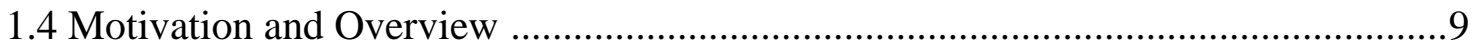

\section{Chapter 2: Coherent Normalization for In Vivo Measurement of Gadolinium in}

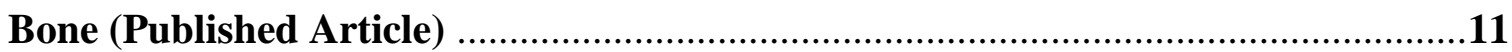

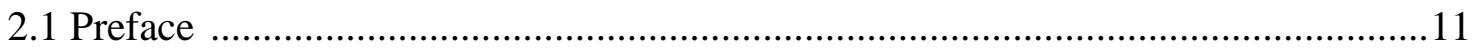

2.2 Article Contents ...............................................................................................13

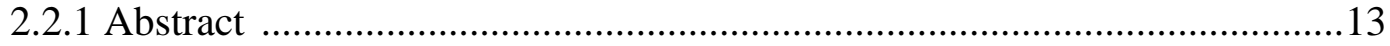

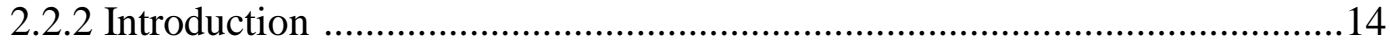

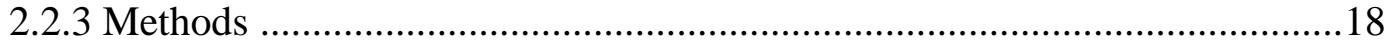

2.2.4 Results and Discussion .....................................................................23

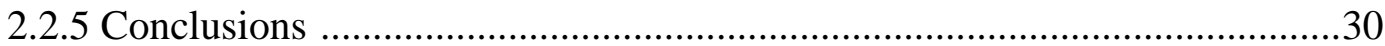

2.2.6 Acknowledgements .........................................................................

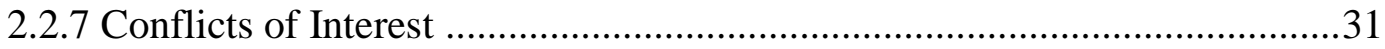

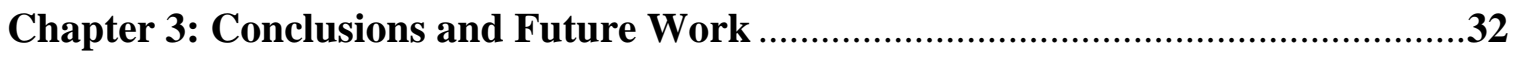

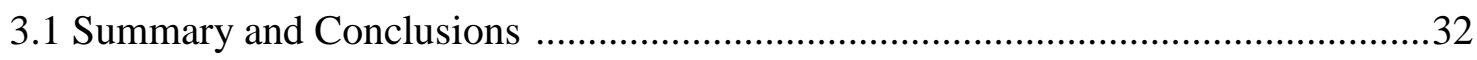

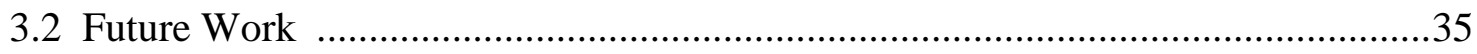

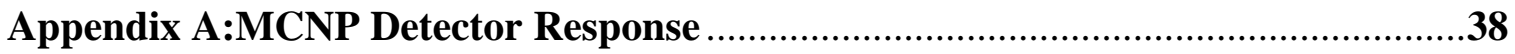

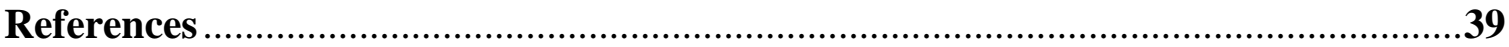




\section{LIST OF FIGURES}

Figure 2.1 MCNP model of the XRF system. a) Top view. b) Isometric view (3D

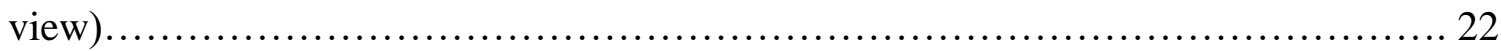

Figure 2.2 Relative contribution of the primary and secondary photon fluence to the Gd K $\mathrm{X}$-ray fluorescence, both with a slope of magnitude $0.0031 \pm 0.0017$, with the scattered fluence having a positive slope, and primary fluence having a negative slope. The statistical uncertainties in these simulation results were less than $1 \%$ .24

Figure 2.3 Gd K x-ray counts normalized to the Cd-109 $88 \mathrm{keV}$ coherently scattered gamma-rays for experimental values for the $120 \mu \mathrm{g} \mathrm{Gd} / \mathrm{g}$ plaster phantom, and simulation values scaled from the $1200 \mu \mathrm{g} \mathrm{Gd} / \mathrm{g}$ plaster phantom

Figure 2.4 A plot demonstrating how the increase of secondary fluence (SFFF) compensates for the differential attenuation of Gd K x-rays and the coherently scattered gamma rays. The product of the secondary fluence fluorescence factor and exponential attenuation ratio results in an insignificant slope of $-0.00075 \pm 0.01000$

Figure A.1 MCNP detector spectrum........................................ 38 


\section{LIST OF SYMBOLS AND ABBREVIATIONS}

$\begin{array}{ll}\text { CCF } & \text { Coherent Conversion Factor } \\ \text { FDA } & \text { Food and Drug Administration } \\ \text { GBCA } & \text { Gadolinium } \\ \text { GDD } & \text { Gadolinium Deposition Disease } \\ \text { HPGe } & \text { High-Purity Germanium } \\ \text { MR } & \text { Magnetic Resonance } \\ \text { MCNP } & \text { Monte Carlo N-Particle } \\ \text { NSF } & \text { Nephrogenic Systemic Fibrosis } \\ \text { OTT } & \text { Overlying Tissue Thickness } \\ \text { PoP } & \text { Plaster of Paris } \\ \text { REE } & \text { Rare Earth Element } \\ \text { XFF } & \text { Secondary Fluence Fluorescence Factor } \\ & \text { X-ray Fluorescence }\end{array}$




\section{Chapter 1: Introduction}

\subsection{Gadolinium Toxicity}

Gadolinium (Gd), the sixty-fourth element in the periodic table, is a rare earth element (REE) that belongs to the lanthanide group of chemical elements [1]. Owing to having seven unpaired electrons in its most outer electronic shell, gadolinium possesses paramagnetic properties which made it the element of choice for magnetic resonance (MR) imaging contrast agents [1], [2]. The presence of gadolinium in tissues shortens the relaxation times of neighboring protons resulting in a signal of higher intensity and enhanced contrast which leads to better disease diagnosis [3].

Since gadolinium is toxic when present in the human body in its free form [4], it has to be bound to a chemically stable chelate complex. Formally known as gadolinium-based contrast agents (GBCA), these agents were first approved for clinical use in 1988 [1]. About $30 \%$ of all MR scans involve the administration of a GBCA. Following the intravenous injection of the contrast agent, it distributes in blood and through extracellular spaces and organs [5]. Preliminary clinical studies indicated that these agents are stable in vivo and that they are completely filtered and excreted through patients' urinary systems, therefore, they are safe [5].

Though it was accepted that GBCAs are safe and are completely eliminated from the body, in 2006 these contrast agents were linked to a progressive and potentially fatal medical condition known as nephrogenic systemic fibrosis (NSF) [6]. NSF was first observed in 
1997 and later described in 2000 in patients who were exposed to GBCA and suffered severe renal dysfunction or end-stage kidney disease [7]. It was found that the excretion half-life of GBCAs in patients with impaired renal function is extended. It is believed that due to the slower clearance rates, these agents are disassociating resulting in deposition of gadolinium in different tissues and organs [6]. Currently, a treatment of NSF does not exist, however, prevention measures have been applied where patients are screened for kidney diseases before being administrated a GBCA. These measures seem to be effective as no NSF cases have been reported after 2009 [8].

Even after the recognition of NSF, GBCAs were perceived as safe drugs as long as they are used in patients with normal renal function and in clinically approved doses [9]. Nonetheless, in recent years, the deposition of gadolinium in bodies of subjects with normal kidney function who were exposed to GBCAs has raised some doubt as to the stability and safety of these agents and it has become an area of concern in the MR imaging community [9]. A study conducted by Kanda et al. compared the MR signal in the brain between two groups, one which received multiple doses of GBCA while the other was exposed to none. The study revealed that the signal in the gadolinium exposed group was significantly higher than the signal in the control group. Also, the signal correlated with number of doses received and was irrespective of patient renal health [3] [9] [10].

In a different study performed by Murata et al., autopsy samples of skin, bone and brain tissue were analyzed. Elevated gadolinium levels were noticed in nine subjects who were exposed to GBCAs and had normal renal function. Results were compared to those of a control group and found to be significantly higher [9]. 
Outcomes of these studies affirm and present strong evidence that the retention of gadolinium is taking place even in patients with normal renal function. Lately, the attention of many research groups has been drawn to studying the adverse effects of the retained gadolinium especially after reports of NSF-like symptoms in individuals with healthy kidney function [11]. Gadolinium Deposition Disease (GDD) is the proposed name used to term gadolinium toxicity in individuals of healthy or near healthy renal system after being exposed to GBCAs [8] [11].

Dr. Richard Semelka, a professor of radiology at the University of North Carolina's school of medicine, led a study that identified 42 patients who have shown evidence of gadolinium retention and suffered from symptoms subsequent to receiving GBCAs. All participants were of healthy renal function [12]. The reported symptoms were similar but not particularly the same as symptoms observed in patients suffering NSF. Reported symptoms included headaches, bone pain, and skin burning sensation. Moreover, skin changes such as thickening and discoloration were observed in many cases. In addition to the aforementioned symptoms, other studies reported brain fog, also known as mentation, besides joint pain and tightness [12].

It is believed that gadolinium disassociates from the chelate in vivo and binds to other endogenous molecules or elements which explains the prolonged clearance rates and long lasting retention [6]. At this time, treatment options of GDD are not available. Chelation therapy has been proposed in which gadolinium is re-chelated in vivo and excreted through the renal system [11]. Such procedure is complex and needs thorough investigation before being approved by the Food and Drug Administration (FDA). 
The proper diagnosis of GDD or any other gadolinium toxicity related condition is reliant on the detection and quantification of the retained amount. Although it is evident that the presence of gadolinium has been confirmed in skin, bone and brain tissue, the bone matrix remains the largest storehouse with concentrations up to 20 times higher than those found in the brain [11].

Detecting the presence of gadolinium can be achieved through methods of tissue investigation and analysis. A drawback of these methods is that they are invasive and painful leaving patients in discomfort. Hence, non-invasive and painless analytical methods such as X-ray fluorescence spectroscopy (XRF) technique, are of vital importance. A robust measuring system can not only determine the amount of retained gadolinium but can also help investigate the efficacy of the chelation therapy if approved. The ability of XRF to detect trace concentrations of gadolinium in bone has been investigated previously, and the method was shown to be feasible [13] [14]. The technique is described in greater detail in the next section of this thesis. 


\subsection{X-ray Fluorescence Spectroscopic Technique}

$\mathrm{X}$-ray fluorescence (XRF) spectroscopy is an analytical technique that enables the quantification of elements of interest in samples [15]. Considered a non-invasive method and of low associated radiation risk, $\mathrm{XRF}$ has been applied in measuring trace concentrations of various toxic and essential elements in vivo [16]. Several XRF systems have been developed to detect elements such as iodine [17], lead [18], cadmium [19], strontium [20], and uranium [21]. Most recently, the feasibility of the method to detect gadolinium [13] and lanthanum [22] in vivo has been confirmed.

The technique relies on creating electronic vacancies in atomic shells. When an atom has a vacancy in one of its inner shells, it is said to be in an excited state. An atom de-excites returning to its ground state when the vacancy is filled with an electron from an outer shell following an electronic transition [23]. The transition results in the emission of a photon. Photons produced via this process are known as fluorescence photons or characteristic Xrays to highlight the fact that emission lines are discrete to each element's atomic structure. A fluorescence photon has an energy that is equivalent to the difference in binding energy between the two shells in which the transition has occurred [23].

The XRF spectroscopic analytical method entails three major steps. At first, photoninduced excitation of the elements under investigation is initiated using either a radioactive source or an X-ray tube. This step is followed by atomic de-excitation and emission of fluorescence photons [24]. The intensity of the fluorescence photons is then registered using a detection system. High-purity Germanium (HPGe) detectors are commonly used 
for XRF analysis due to their high resolution. The intensity of the produced fluorescence fluence is proportionally related to the amount of the analyte present in the sample [24]. The detected signal is converted to concentration through calibration with appropriate calibration standards.

Although the XRF spectroscopic technique is a robust method for detecting elements in vivo, correlating the fluorescence signal intensity to the actual amount of the analyte can be challenging [24]. This is due to factors affecting the XRF signal. For instance, in a tibial $\mathrm{XRF}$ measurement, the measured fluorescence intensity is a function of soft tissue thickness covering the tibia where the measurement is taking place, this is also acknowledged as the measurement site. Other geometrical factors such as the size and shape of the bone can have an impact on the signal [18] [24] [25] [26].

In order to achieve a quantification that is only dependent on the concentration of the element under investigation regardless of all the other factors, corrections must be applied to account for any tissue attenuation and geometrical effects. In the work published by Somervaille et al. on the in vivo measurement of lead in bone, it was concluded that the need for extensive correction factors can be eliminated contingent upon the validity of the coherent normalization method [18]. The coherent normalization process as a correction procedure is described in further detail in the next section of this thesis. 


\subsection{Coherent Normalization Method}

With a low associated radiation risk, X-ray fluorescence (XRF) spectroscopy remains a useful and effective method to determine concentrations of elements in vivo [16]. The robustness of the method can be reduced due to aspects that make the absolute quantification of elemental concentration difficult to achieve [18] [26]. When analyzing a sample-containing analyte via XRF, the detected X-ray signal must be correlated to the amount of the element in the measured volume under investigation [24]. However, other factors can affect the recorded signal.

In conducting bone in vivo XRF studies, factors other than the quantity of the analyte can have an effect on the number registered X-ray fluorescence peak [24]. The thickness of soft tissue covering the measurement site, which is also known as overlying tissue thickness (OTT), attenuates the photon fluence making the detected XRF signal a function of tissue thickness. Other interpatient variabilities such as the size and shape of the bone influence the measurement [18] [21] [25]. Furthermore, the geometry of the measurement, primarily the source-to-sample distance, can be of influence.

For the purpose of taking into account all the factors impacting the measurement, a detailed knowledge of the measurement site such as the size and the shape of the bone as well as OTT becomes necessary. This can be accomplished by conducting independent examinations of the measurement site, such as taking radiographs [26]. In spite of that, it was shown that the use of the coherently scattered photon peak area as a normalizing factor 
can produce a measurement that is independent of all the circumstances mentioned earlier [18] [26].

The use of the coherent normalization as a correction method involves taking the ratio of the detected X-ray peak counts and the coherent peak. The resultant ratio is known as the coherent ratio [27]. A successful use of the coherent normalization method accomplishes the goal of having a measurement that is independent of any geometrical factors or interpatient variabilities without the need to perform any radiographs, as a result patient radiation dose is reduced, and practical issues regarding obtaining radiographs are removed [18] [26]. Additionally, the coherent ratio is related to the amount of element being measured without the dependency on the source activity, detector dead time and the acquisition interval [25] [27] [28].

Because the coherent ratio is dependent on the size of the coherent peak, it is important that the coherent scattering profile between the calibration standard and the actual bone mineral are the same. If they are not, an additional correction is needed to account for the differences in the extent of coherent scatting between the two matrices. Such factor is known as the coherent conversion factor $(\mathrm{CCF})$ and is defined as the of the ratio relativistic form factors of the calibration standard and bone mineral [29] [30]. 


\subsection{Motivation and Overview}

The potential long-term effects of the retained gadolinium in patients exposed to gadolinium-based contrast agents are concerning. Detecting the presence of retained gadolinium is important to assist in diagnosing medical conditions related to gadolinium toxicity [8]. Knowing that bone is the largest deposition site for gadolinium, the X-ray fluorescence (XRF) spectroscopic technique has been previously investigated to detect gadolinium in bone tissue [13] [31].

The XRF method is painless, non-invasive and of low radiation risk, however, geometrical factors and other interpatient variabilities can cause the measurement to be misperceived. A successful application of the coherent normalization method results in an XRF measurement that is only a function of the amount of the element present in the measured volume. The ability of the coherent normalization method to correct for interpatient variability is conditional on four criteria that have been satisfied in the lead measurement system described by Somervaille et al. [18], however, in the case of gadolinium two of the four criteria are not met. These criteria are discussed in more detail in Chapter 2 of this thesis. Photons emitted by the ${ }^{109} \mathrm{Cd}$ excitation source are approximately $37.7 \mathrm{keV}$ higher than the K-edge energy of gadolinium, meaning that X-ray fluorescence is being produced by primary photons as well as photons that have scattered but still have sufficient energy to induce the photoelectric effect in the K-shell. Additionally, fluorescence photons emitted by gadolinium suffer greater attenuation in tissue compared to the $88.034 \mathrm{keV}$ coherently scattered photons from the source. Hence, a careful investigation of this method is crucial. 
This work intended to investigate the hypothesis that although not all four coherent normalization criteria are met, the method can still be effective in correcting for varying OTT due to factors that counteract the differential attenuation of fluorescence and coherently scattered photons, this has been confirmed for an XRF system previously developed to detect bone uranium. To test the hypothesis, a Monte Carlo based investigation of the validity of the coherent normalization method as a correction procedure for the measurement perturbation factors discussed above was performed.

The methods and results of this study, published in the journal Physiological Measurement, are presented in greater detail in Chapter 2. The coherent normalization procedure was demonstrated effective over a large tissue thickness ranging between 0 and $12.2 \mathrm{~mm}$ representative of a typical adult population. Thesis conclusions and future work are presented in Chapter 3. 


\section{Chapter 2: Coherent Normalization For In Vivo Measurement of Gadolinium in Bone (Published Article)}

\subsection{Preface}

This chapter presents an article that was published in the journal PhysiologicalMeasurement. The article was authored by two co-first authors who contributed equally to the work; Ms. Michelle Lindsay Lord as well as the author of this thesis. The experimental work was conducted by Ms. Lord under the supervision of Drs. McNeill and Chettle, and the simulation work was performed by the author of this thesis under the supervision of his direct supervisor, Dr. James Gräfe. All co-authors contributed to editing the manuscript.

The validity of the coherent normalization method to correct for variation in overlying tissue thickness for a ${ }^{109} \mathrm{Cd}$ based $\mathrm{XRF}$ bone gadolinium measurement system was investigated through experimental work and Monte Carlo simulations. The article outlines evidence from the literature that supports the significance and the need for this investigation.

With regards to the simulation work, the latest available version of the general-purpose radiation transport code, Monte Carlo N-Particle (MCNP6.1.1) was used to produce a realistic model of the ${ }^{109} \mathrm{Cd}$ based $\mathrm{X}$-ray fluorescence system including the cloverleaf detection system that was used in the experiment. The significance of the simulation work lies in the ability to provide a methodical explanation of the method's feasibility through tallying the interactions induced by the two fluence components, scattered and primary, 
separately, and defining the Secondary Fluence Fluorescence Factor (SFFF). This is discussed in greater detail in the following section. Copyright permission was granted to reproduce the article as part of this thesis. 


\subsection{Article Contents}

(C) Institute of Physics and Engineering in Medicine. Reproduced with permission. All rights reserved.

\section{Coherent normalization for in vivo measurements of gadolinium in bone}

\section{Z Keldani' ${ }^{1,4}$, M L Lord ${ }^{2,4}$, F E McNeill ${ }^{3}$, D R Chettle³, J L Gräfe ${ }^{1}$}

\subsubsection{Abstract}

Recent evidence of gadolinium $(\mathrm{Gd})$ deposition in bones of healthy individuals who have previously received Gd-based contrast agents (GBCAs) for MRI, has led to a demand for in vivo measurement techniques. The technique of $\mathrm{x}$-ray fluorescence (XRF) provides a low risk and painless method to assess Gd deposition in bone, and has the potential to be a useful clinical tool. However, interpatient variability creates a challenge while performing in vivo measurements. We explored the use of coherent normalization, which involves normalizing the $\mathrm{Gd} \mathrm{K} \mathrm{x}$-rays to the coherent scattered $\gamma$-ray from the excitation source, for bone Gd measurements through a series of phantom-based experiments and Monte Carlo simulations. We found coherent normalization is able to correct for variation in overlying

\footnotetext{
${ }^{1}$ Department of Physics, Ryerson University, Toronto, M5B 2K3, Canada

${ }^{2}$ Radiation Sciences Graduate Program, McMaster University, Hamilton, L8S 4L8, Canada

${ }^{3}$ Department of Physics and Astronomy, McMaster University, Hamilton, L8S 4L8, Canada

${ }^{4}$ Authors contributed equally to this work
} 
tissue thickness over a wide range $(0-12.2 \mathrm{~mm})$. The $\mathrm{Gd}$ signal to coherent signal ratio is independent of tissue thickness for both experiments and Monte Carlo simulations. Coherent normalization has been demonstrated to be used in practice with normal healthy adults to improve in vivo bone $\mathrm{Gd}$ measurements.

\subsubsection{Introduction}

The routine use of gadolinium-based contrast agents (GBCAs) for MRI in hospitals worldwide is currently being questioned due to recent evidence of gadolinium (Gd) deposition in tissues of healthy individuals [10][32][33][34][35]. Not only has Gd been identified in tissues, such as brain and bone, symptoms associated with Gd toxicity are now being recognized in some individuals who have previously received GBCAs [8][36]. For this reason, the safety of GBCAs is currently a major concern in the MRI community.

Bone tissue has been shown to serve as a major storage site for Gd in the body [9][37][38][39]. The free $\mathrm{Gd}^{3+}$ ion is one of the largest competitive inhibitors for the $\mathrm{Ca}^{2+}$ ion, and is therefore believed to replace calcium in bone mineral [40]. Currently Gd levels in bone can only be measured through invasive techniques, which are inconvenient and painful, and in fact, many of the literature data of $\mathrm{Gd}$ in bone stem only from autopsy samples. X-ray fluorescence (XRF) is a non-invasive, painless technique that can be used to detect Gd in bone through an in vivo measurement. The XRF system developed in our lab has been shown to be successful in measuring Gd in bone phantoms and autopsy bone samples, and is capable of carrying out 30-minute tibial measurements on volunteers, 
involving a low dose of $0.13 \mu \mathrm{Sv}$ per measurement [31]. The measurement involves the subject placing their leg in front of a Cd- 109 excitation source that is mounted on the face of a high purity germanium detector, which is described in further detail by Lord et al. [31].

Conducting human tibial measurements using the XRF system involves interpatient variability, with respect to the excitation source to measurement site distance, the size of the bone, and the depth of overlying tissue. Coherent normalization involves normalizing the measured $\mathrm{K}$ x-ray peak areas to the coherent peak area of the excitation source, and has been successfully used with previous XRF systems to correct for this interpatient variability.

Somervaille et al. investigated the use of coherent normalization for a similar XRF system consisting of a $\mathrm{Cd}-109$ excitation source to measure lead $(\mathrm{Pb})$ in human tibia [18]. For coherent normalization to be valid, the system must satisfy four criteria:

(1) the $\mathrm{Pb} \mathrm{K} \mathrm{x}$-rays and coherent scatter signals must be produced from the same fluence,

(2) both signals must be created from the same location in the sample,

(3) both signals must have the same angular distribution,

(4) both signals must be attenuated similarly on their way back to the detector.

The system described by Somervaille et al. for bone- $\mathrm{Pb}$ measurements satisfies most criteria for coherent normalization. Firstly, since the Cd-109 $\gamma$-ray energy is close to the Kedge of $\mathrm{Pb}$, the $\mathrm{Pb} \mathrm{K}$ x-rays are mostly produced from the primary excitation $\gamma$-ray, and not from secondary fluence such as Compton scattered photons. Secondly, the coherent 
scattering cross section for the Cd-109 $88 \mathrm{keV}$ excitation energy in cortical bone is approximately 28 times larger than the cross section for soft tissue, meaning both the $\mathrm{K}$ xrays and coherent $\gamma$-rays are produced in bone. Thirdly, the $\mathrm{K}$ x-rays are produced isotropically while the coherent scattering is not isotropic. However, the differential coherent scattering cross section is relatively constant over the angles encountered in the backscatter geometry at an energy of $88 \mathrm{keV}$. Lastly, the difference in attenuation between the $\mathrm{Pb} \mathrm{K} \mathrm{x}$-ray $(72.8 \mathrm{keV})$ and the coherent $\gamma$-ray $(88 \mathrm{keV})$ through bone and tissue results in only a small difference in measured signals due to the similar energies [18].

When comparing the XRF system used by Somervaille et al. to our XRF system, there is one major difference: we are detecting Gd instead of $\mathrm{Pb}$. The excitation source energy of $88 \mathrm{keV}$ and $180 \mathrm{o}$ geometry remains the same, causing criteria (2) and (3) to be satisfied for the Gd XRF system. It is the large difference in energies of the Gd K X-rays (43 keV) and coherent $\gamma$-rays $(88 \mathrm{keV})$ that leads to questioning the feasibility of coherent normalization for human measurements. For criterion (1), the $88 \mathrm{keV}$ excitation energy is $37.7 \mathrm{keV}$ above the Gd K-edge (50.24 keV). If the excitation $\gamma$-ray undergoes Compton backscatter, resulting in the maximum energy loss for a single Compton event, the scattered x-ray will still be $15.2 \mathrm{keV}$ above the Gd K-edge. For this reason, it is very likely that Gd $\mathrm{K}$ x-rays are being produced by secondary fluence from Compton scatter. Therefore, coherent $\gamma$-rays are produced by the primary fluence of the excitation $\gamma$-rays, where Gd K x-rays are produced by both primary and secondary fluence. The difference in energy between the Gd $\mathrm{K}$ x-rays and coherent $\gamma$-rays lead to a differential attenuation through bone and tissue, causing criterion (4) to be left unsatisfied. 
Although criteria (1) and (4) are not satisfied due to the lower Gd K x-ray energy, certain counteracting factors have deemed coherent normalization feasible in XRF systems where not all four criteria are met. An XRF system by O'Meara et al. using a Co-57 excitation source to measure uranium (U) in bone did not meet criteria (1) and (4) due to the excitation energy of $122 \mathrm{keV}$ being $6.5 \mathrm{keV}$ above the U K-edge. Coherent normalization was not expected to work for this system, yet a Monte Carlo simulation showed that the ratio of $\mathrm{U}$ $\mathrm{K} \mathrm{x}$-rays to coherent $\gamma$-rays remains constant over a large range of overlying tissue, indicating the normalization is valid. As the overlying tissue thickness increases, the primary fluence from the excitation $\gamma$-ray decreases, thus de- creasing both the XRF signal and coherently scattered signal. However, the secondary fluence from Compton events increases, which counters the decrease in primary fluence contributing to the XRF signal and may compensate for the differential attenuation of the excitation $\gamma$-ray and lower energy XRF signal. This effective cancellation could be the reason for coherent normalization functioning for this system, and could be a contributing factor in the Gd XRF system as well [41]. Sufficient countering influences were also present in an XRF system using a Co-57 source to mea- sure lead in bone. The change in K x-ray signal to coherent signal ratio with overlaying tissue was investigated by experiment and Monte Carlo simulation and indicated that the normalization procedure is acceptable for this system [42].

Given the examples of valid normalization on past XRF systems, it is possible that divergences from the four criteria will result in counteracting factors that deem normalization a valid procedure for Gd measurements conducted on our XRF system. In 
this paper, we investigate the use of coherent normalization through experiment and Monte Carlo simulation. We use the Monte Carlo code MCNP6 [43] to simulate the components of Gd K x-ray production and subsequent attenuation to understand countering influences contributing to the validity of coherent normalization.

\subsubsection{Methods}

\subsubsection{Experimental Measurement}

A set of tibia bone phantoms was constructed using Plaster of Paris $(\mathrm{CaSO} 4 \cdot 2 \mathrm{H} 2 \mathrm{O})$ to represent bone, for previous experiments that explored the feasibility of using our XRFsystem to detect Gd in bone [13][31]. The bone phantoms are anthropomorphic to a human tibia, in the shape of a cylindrical shaft with a diameter of $28.0 \mathrm{~mm}$ and length of $115 \mathrm{~mm}$. Gadolinium solution, prepared from gadolinium oxide ( $\mathrm{Gd} 2 \mathrm{O} 3)$ dissolved in dilute nitric acid, was added to the plaster mixture to create phantoms of varying Gd concentrations. The bone phantom with a concentration of $120 \mu \mathrm{g} \mathrm{Gd} / \mathrm{g}$ plaster was used for the experiments presented in this article, since this is the highest concentration and will therefore produce the strongest Gd signal in measurements.

The XRF system used is a "cloverleaf" high purity germanium detector (HPGe) from Canberra Industries (GL0210R/S), consisting of 4 smaller detectors in a cloverleaf formation (Fig.1). Each HPGe detector is $16 \mathrm{~mm}$ in diameter and $10 \mathrm{~mm}$ thick, and attached to its own set of pulse processing electronics, including a preamplifier (Canberra 2002CP) and digital pulse processing system (Canberra DSA 1000). Data are collected on four 
separate spectra using Genie 2000 Gamma Analysis Software from Canberra. The four spectra are then exported for further analysis of the $\mathrm{Gd}$ and Coherent signals using MATLAB.

A Cd-109 excitation source, with an activity ranging from 4.11-4.04 GBq at the time of these experiments, was mounted to the face of the detector. The Cd-109 source emits excitation $\gamma$-rays of $88 \mathrm{keV}$ and silver $\mathrm{x}$-rays in the range of $22-25 \mathrm{keV}$. The source was enclosed in a tungsten collimator with a copper face to filter out the silver x-rays [13]. The phantom was placed at a close distance of $1 \mathrm{~mm}$ directly in front of the source to create a 180-degree geometry. Measurements were conducted on the bone phantom for 24 hours live-time, with tissue equivalent material (Solid Water ${ }^{\circledR}$ ) of thicknesses of $3.3 \pm 0.1$ $\mathrm{mm}, 4.0 \pm 0.1 \mathrm{~mm}, 7.5 \pm 0.1 \mathrm{~mm}$, and $12.2 \pm 0.1 \mathrm{~mm}$, to investigate how the Gd signal to coherent signal ratio changes with tissue thickness. A longer measurement time was chosen over a realistic in vivo measurement of 30 minutes, to lower measurement uncertainties.

For a measurement, each spectrum was analyzed by fitting the two $\mathrm{Gd} \mathrm{K} \alpha$ peaks at 42.3 and $43 \mathrm{keV}$, as well as the coherent peak at $88 \mathrm{keV}$. The method of Non-Linear Least Squares was used in MATLAB to fit the Gd peaks with a double Gaussian function and a polynomial background, since the peaks were located on the side of another feature on the spectra. The coherent peak was fit using a single Gaussian and linear background, as this peak did not interfere with any other features on the spectra. All peak fits resulted in $\chi^{2}$ values of $1.0 \pm 0.20$. Peak areas were then calculated from the Gaussian constants obtained from the fit, and the ratio of Gd signal to coherent signal was calculated and plotted as a function of overlying tissue equivalent material. 


\subsubsection{Monte Carlo Simulations}

The latest release of the general purpose Monte Carlo N-Particle (MCNP) radiation transport code, MCNP6, was used in this work. MCNP6 was first introduced by Los Alamos National Laboratories in 2012, and is the merger of capabilities of MCNP5 and MCNPX [44]. Besides incorporating capabilities of earlier MCNP versions, MCNP6 offers several newly added features. Those of significance to this work are enhanced photon form factors and complete atomic relaxation processes [45]. The enhanced code's performance is based on the introduction of the extended Evaluated Nuclear Data File (ENDF/B VI.8) as well as the improved transport methods [45]. ENDF/B VI.8 contains extended form factor functions that cover a larger energy and angular range compared to previous releases. Previous versions could not adequately model coherent scattering for photon energies above $74 \mathrm{keV}$ [46]. Additionally, a correct logarithmic method replaced the older linear interpolation of form factors. These updates lead to a comprehensive representation of coherent and incoherent scatter events, which is significant for modelling backscattering experiments. The updated data can be requested using the $.12 \mathrm{p}$ photon transport table in the material card of the input file [45]. MCNP6 is able to model complete atomic relaxation processes including fluorescent photons and Auger electrons down to the energy cut-off of each. Previous MCNP versions considered only K-shell transitions as well as weighted Lshell transitions. The extended library includes data in energy ranges as low as $1 \mathrm{eV}$ allowing for more comprehensive calculations to be done. 


\subsubsection{Monte Carlo model of the Gd K-XRF system}

A realistic model of the Cd-109 based XRF system including the clover-leaf HPGe detection system, collimator, phantom and Solid Water ${ }^{\circledR}$ was created in MCNP6 [31]. This is shown in Figure 2.1. The detector dimensions were extracted from the manufacturer's drawings and all other dimensions were chosen to reproduce the experimental set-up. The Cd-109 source was modelled as a simple point source inside the custom tungsten collimator. We modelled the experiment exactly, except we did not include the $1 \mathrm{~mm}$ thick overlying plastic of the bone phantoms. The Plaster of Paris (PoP) phantom and Solid Water ${ }^{\circledR}$ were modelled as non-concentric cylinders. In order to improve the statistical results in the Gd peak regions of interest, we modelled the bone phantom to contain a Gd concentration of $1200 \mu \mathrm{g} \mathrm{Gd} / \mathrm{g}$ plaster. The Monte Carlo results were then scaled down to $120 \mu \mathrm{g} \mathrm{Gd} / \mathrm{g}$ plaster, to match the experimental results. One concern with this approach would be the small contribution of the extra Gd to the coherent scattering within the phantom. We investigated this by turning off coherent scattering in Gd only, which is accomplished by not calling on the new $.12 \mathrm{p}$ photon interaction tables for $\mathrm{Gd}$ in the material card, and found no significant effect on the coherent scattering from the phantom. 


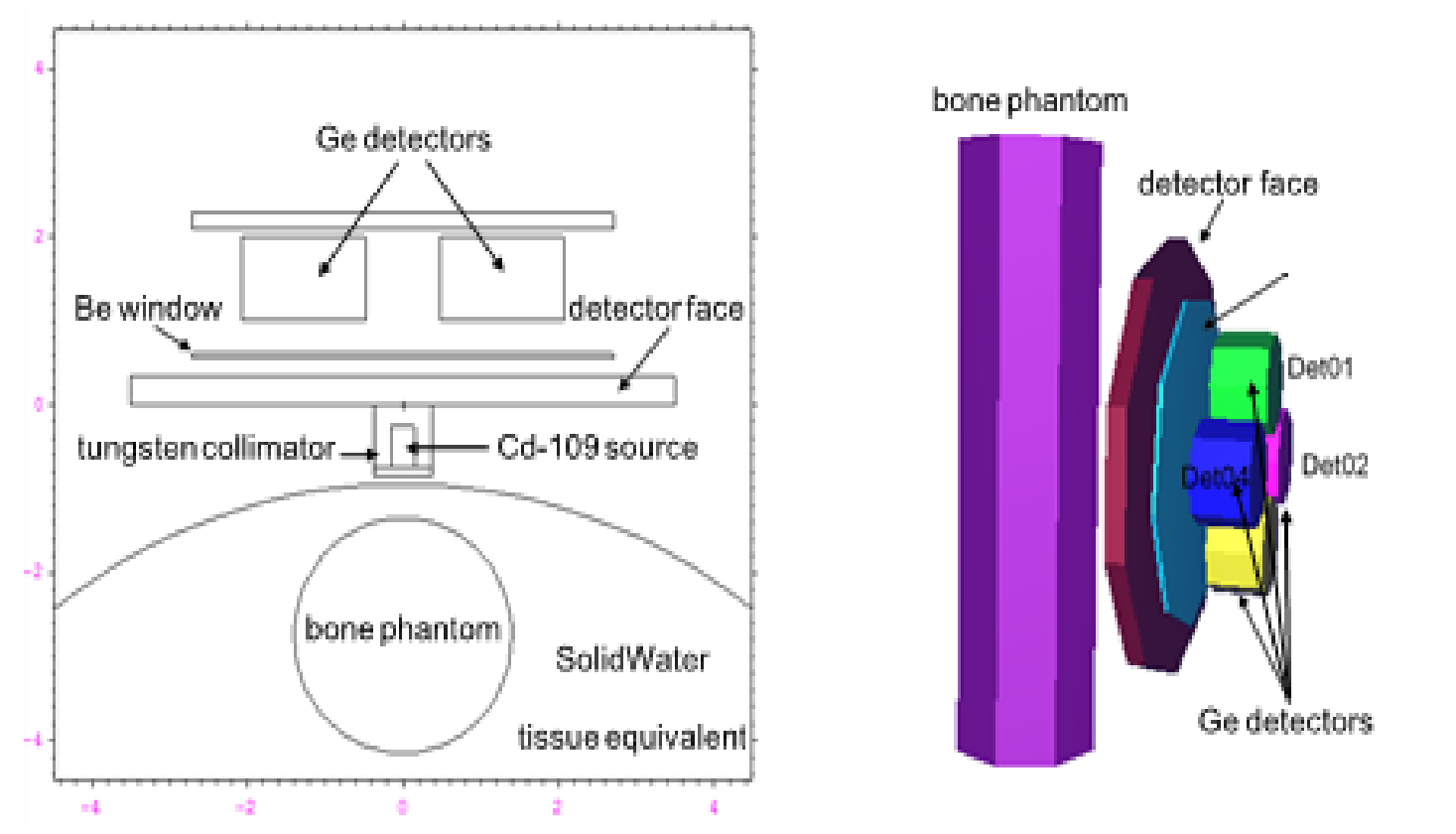

Figure 2.1 MCNP model of the XRF system. a) Top view. b) Isometric view (3D view).

The pulse height tally, F8, was used to simulate the detector response in order to determine the Gd K x-ray peak intensities as well as the backscattered coherent peak intensity. Only data from one of the four cloverleaf detectors was tallied in the simulations. Although there is limited detector-to-detector variation, we compared the same detector (Det02) in both the simulation and experimental results. As mentioned earlier, photons emitted by Cd-109 are approximately $37.7 \mathrm{keV}$ higher than the K-edge energy of Gd. That means, scattered photons of energies higher than the K-edge energy of Gd can still undergo a photoelectric effect and produce characteristic x-rays. Hence, the production of Gd x-rays is caused by two fluence components; the primary fluence, consisting of non-scattered photons, and a secondary fluence which includes Compton scattered photons. The code was used to determine the relative contribution of the primary and secondary components to the $\mathrm{Gd} \mathrm{K}$ 
X-ray fluorescence. The FM4 tally multiplier card was used to tally the photoelectric reaction rate per unit volume for the primary and secondary fluence components respectively.

$$
\begin{aligned}
& R_{\text {primary }}(E)=n \int_{87.999}^{88.034 \mathrm{keV}} \Phi(E) \sigma(E) d E \\
& R_{\text {secondary }}(E)=n \int_{50.2}^{87.999 \mathrm{keV}} \Phi(E) \sigma(E) d E
\end{aligned}
$$

Where $\mathrm{n}$ is the number of $\mathrm{Gd}$ atoms per unit volume. Equation 1 is written as an integral, but it essentially represents the reaction rate for the mono-energetic $88.034 \mathrm{keV}$ primary photons with Gd. Equation 2, represents the integration of the photon fluence spectrum, $\Phi$ $(E)$, over the energy dependent cross section $\sigma(E)$. The integration limits span energies above the K-edge of $\mathrm{Gd}$ to an energy of $87.999 \mathrm{keV}$ (an energy we arbitrarily used to represent the cut-off between scattered and primary photons in the simulation). As in the experiments, the simulations were run for varying Solid Water ${ }^{\circledR}$ thicknesses ranging between 0 to $12.2 \mathrm{~mm}$ while keeping the distance between the measurement site and the collimator constant at $1 \mathrm{~mm}$.

\subsubsection{Results and Discussion}

The relative contribution of the primary and secondary components of the photon fluence to Gd K x-ray fluorescence interactions in the bone phantoms as a function of tissue thickness, modelled by Monte Carlo simulations, is shown in Figure 2.2. The secondary fluence contributes a significant amount to the fluorescence of Gd. In fact, it is the dominant 
contribution. The Monte Carlo code cannot distinguish between interactions resulting in K-shell fluorescence and higher shells. Since photons with energy above the K-edge, by default possess enough energy to fluoresce the higher shells, these will be included in the integration results of equations 1 and 2 . If the photon energy exceeds the K-edge, the relative amount of fluorescence events contributing to K-shell events compared to higher shell events is a fixed probability, and the ratio is typically about 4:1 [47], favouring the K-shell. Since both integrations in equations 1 and 2 only include photons with energies above the K-edge of $\mathrm{Gd}$, by taking relative interaction rates, the K-shell to L-shell and higher shell fluorescence emission probability ratio will factor out.

The proportion of $\mathrm{x}$-rays produced by the secondary fluence increases with tissue thickness, with the opposite being true for the primary fluence component. However, both components share the same degree of change (magnitude of slope) i.e. the rate of change of each component's contribution as a function of tissue thickness. The scattered fluence increase with thickness, while the primary fluence decreases with thickness, as expected. 


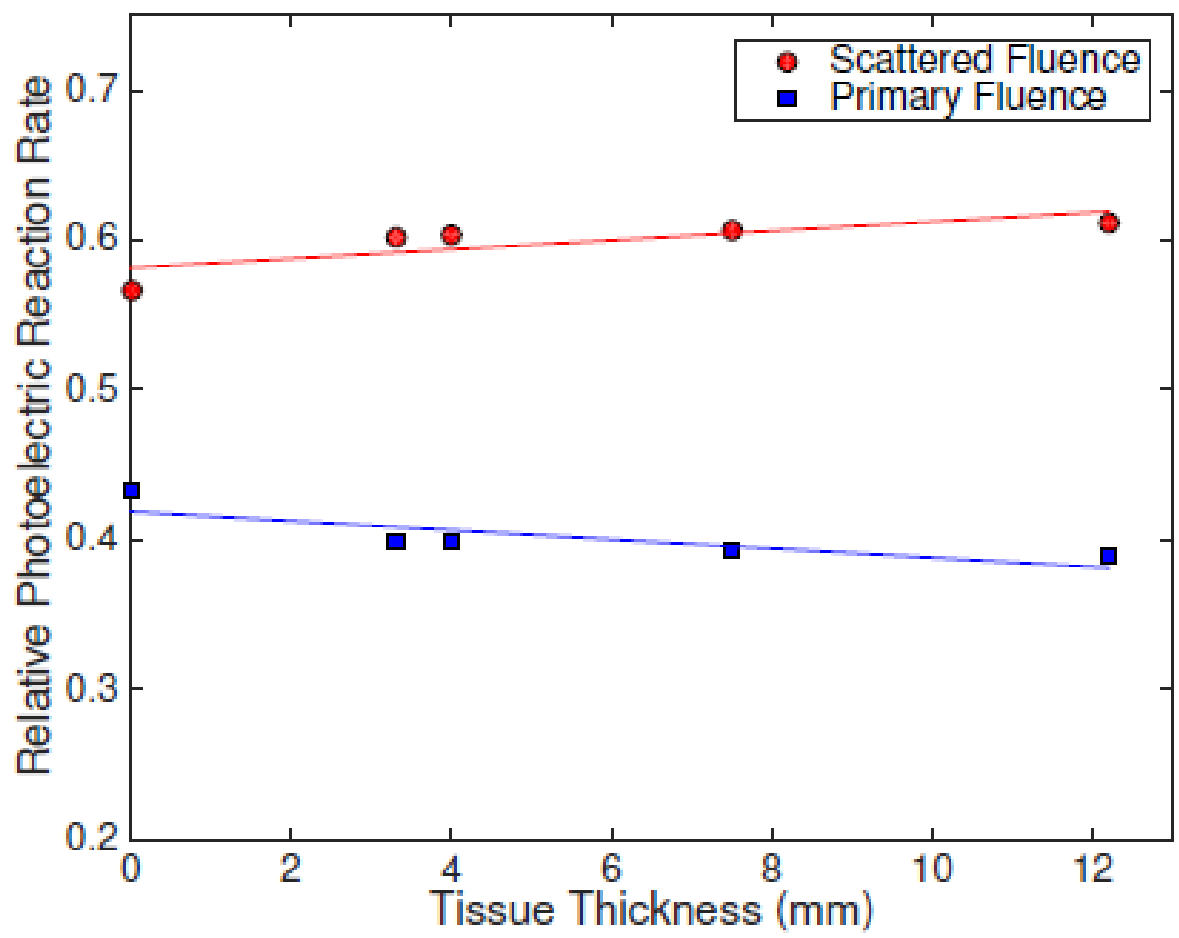

Figure 2.2 Relative contribution of the primary and secondary photon fluence to the $\mathrm{Gd} \mathrm{K}$ x-ray fluorescence, both with a slope of magnitude $0.0031 \pm 0.0017$, with the scattered fluence having a positive slope, and primary fluence having a negative slope. The statistical uncertainties in these simulation results were less than $1 \%$.

For the $1200 \mu \mathrm{g} \mathrm{Gd} / \mathrm{g}$ phantom Monte Carlo simulations, the coherent normalization of $\mathrm{Gd}$ $\mathrm{K}$ x-ray signal to coherently scattered signal as a function of tissue thickness results in a line with a slope of $-0.0053 \pm 0.0040$ (one standard deviation). The slope is within 1.3 uncertainties of zero and is therefore not significant at the $95 \%$ confidence level. This suggests the signal ratio is independent of tissue thickness, and that the normalization method is acceptable for tissue thicknesses in the range of $0-12.2 \mathrm{~mm}$. This relationship 
is shown in Figure 2.3, and illustrates the relationship between Gd x-ray-to-coherent ratio and tissue thickness. The average ratio is $0.717 \pm 0.025$, suggesting the normalized signal varies by $3.5 \%$ within one standard deviation. This is acceptable considering other uncertainties involved in the in vivo measurement procedure. For tissue thicknesses ranging from $3.3-7.5 \mathrm{~mm}$, the $1200 \mu \mathrm{g} \mathrm{Gd} / \mathrm{g}$ phantom simulation data results in an insignificant slope of $-0.0043 \pm 0.014$. Since the average tissue thickness over an adult tibia is approximately 4-5 $\mathrm{mm}$ [48], the insignificant slope between 3.3-7.5 $\mathrm{mm}$ suggests coherent normalization is especially useful for the average healthy individual.

Experimental data are displayed in Figure 2.3, which result in a line with a slope of zero within statistical uncertainties $(0.0021 \pm 0.0059)$, and an average ratio of $0.749 \pm 0.019$, suggesting the normalized signal varies by $2.5 \%$ within one standard deviation. As previously mentioned, all data illustrated in Figure 2.3 are from 1 of 4 cloverleaf detectors (Det02), as data from each of the cloverleaf detectors are statistically the same. Experimental measurements resulted in an insignificant slope for tissue thicknesses ranging from $0-12.2 \mathrm{~mm}$, suggesting coherent normalization is valid over the entire range. Coherent normalization was also deemed valid over this tissue thickness range for the 1200 $\mu \mathrm{g} \mathrm{Gd} / \mathrm{g}$ phantom simulations, since the slope was insignificant at the $95 \%$ confidence level. There is a slight tendency towards a negative slope for the Monte Carlo simulations of the coherent normalization. This will be discussed below in comparison to the simulation, which separates each interaction into components. It should also be noted that we have made every attempt to model the detector positioning, components, and materials as exact to the experiment as possible, however, we have not made an attempt to benchmark 
the detection efficiency. Despite these limitations, we argue that it is remarkable to have the average coherent normalization ratio agree with the experiment ratio, as these numbers are not significantly different $(\mathrm{p}=0.43)$.

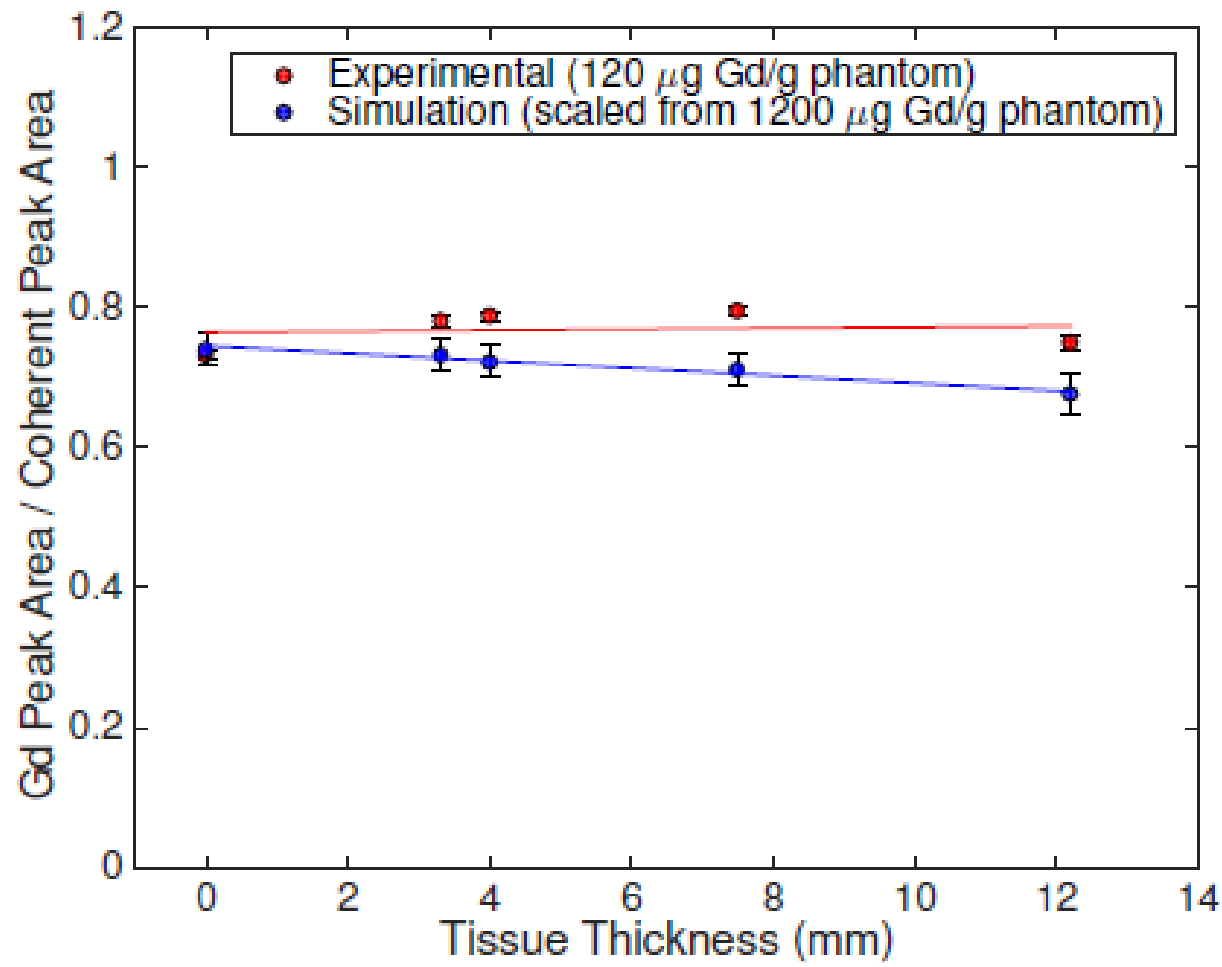

Figure 2.3 Gd K x-ray counts normalized to the Cd-109 $88 \mathrm{keV}$ coherently scattered gamma-rays for experimental values for the $120 \mu \mathrm{g} \mathrm{Gd} / \mathrm{g}$ plaster phantom, and simulation values scaled from the $1200 \mu \mathrm{g} \mathrm{Gd} / \mathrm{g}$ plaster phantom.

As the overlying tissue thickness is increased there is an increase of secondary Compton scattered photons. As photons lose energy due to these scattering events they become closer in energy to the K-edge energy of Gd, meaning that they have a higher probability (larger 
cross section) of inducing a photoelectric effect. Both Gd x-rays and coherently scattered photons are attenuated by the tissue. Gd x-rays of approximately $43 \mathrm{keV}$, experience a higher attenuation compared to $88 \mathrm{keV}$ coherently scattered photons. However, this differential attenuation is effectively counteracted by the increase in secondary fluence, which we will refer to as the Secondary Fluence Fluorescence Factor (SFFF). The SFFF is the ratio of the total photoelectric effect reaction rate (sum of equations 1 and 2) to the primary reaction rate (equation 1) in the bone phantoms, and represents the proportion of fluorescence emission induced by the secondary pho- ton fluence. SFFF does not rely on the angular distribution contained in the cross section tables for any specific element. The normalized secondary fluence fluorescence factor (normalized to zero overlying tissue thickness to demonstrate the impact of tissue scattering) is plotted in Figure 2.4. In addition, the ratio of the differential exponential attenuation of the approximately $43 \mathrm{keV} \mathrm{Gd} \mathrm{x-rays}$ to the $88 \mathrm{keV}$ as a function of Solid Water ${ }^{\circledR}$ thickness was also plotted. The product of these two components counteract each other, producing a relatively flat line with no significant slope. This method does not rely on the differential coherent scattering cross sections as a function of scattering angle (ie. the angular dependence) as would the simulation of the detector measured spectrum from which the results of Figure 2.3 are obtained. As coherent scattering with all elements over all energies is a new addition to the MCNP6 code, there may be some discrepancies inherent in the code, and this is an area of future research we intend to conduct. Furthermore, any deviations in the Solid Water ${ }^{\circledR}$ composition used in the experiment and Monte Carlo work could result in different 
coherent scattering within the Solid Water ${ }^{\circledR}$, which may influence the coherent scattering ratio.

We have shown through experiment and Monte Carlo simulation that the coherent normalization procedure, originally designed for the bone- $\mathrm{Pb}$ system [18] works reasonably well for the Gd system. The coherent normalization is valid for varying body types and overlying tissue thicknesses up to $12.2 \mathrm{~mm}$ for the bone-Gd system. The Monte Carlo simulation of the full experiment demonstrates a slight trend to a negative slope compared to experimental work, however over the typical range of overlying tissue thickness for healthy adults there is no trend in the coherent normalization. The simulation involving the components contributing to the coherent normalization removes any inherent unknown factors in the data tables utilized in the code, and provides a theoretical explanation of the experimental work demonstrating the effectiveness of the coherent normalization. Some limitations of our study are the use of Solid Water ${ }^{\circledR}$ as a tissue equivalent substitute at these energy ranges. It is difficult to match soft tissue composition, overlying fat or skin, at the energy range of interest for our work and this is obviously an area of future research. We have not investigated the coherent normalization as function of distance from the source, however, the design of our measurement system minimizes any potential participant movement. 


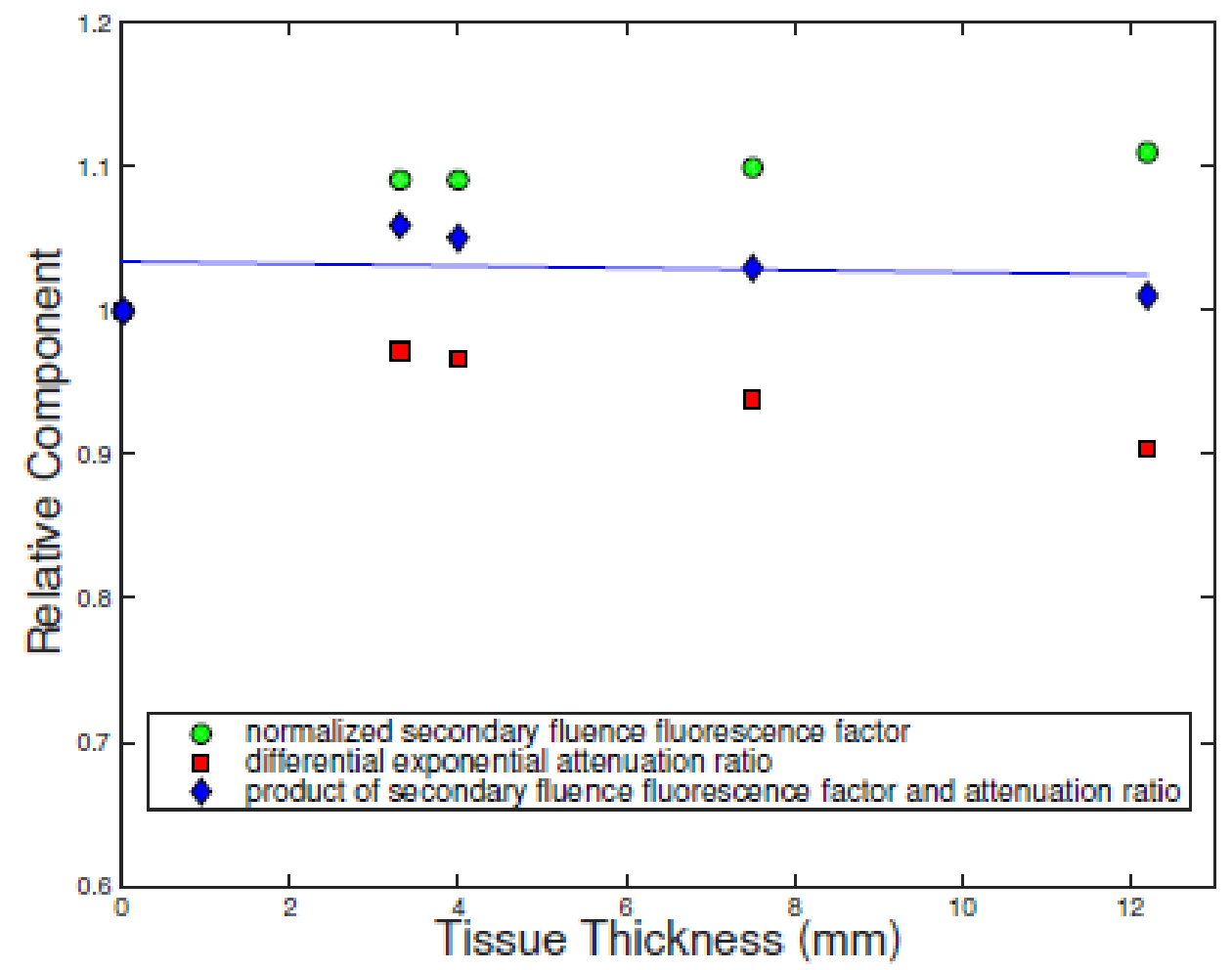

Figure 2.4 A plot demonstrating how the increase of secondary fluence (SFFF) compensates for the differential attenuation of Gd K x-rays and the coherently scattered gamma rays. The product of the secondary fluence fluorescence factor and exponential attenuation ratio results in an insignificant slope of $-0.00075 \pm 0.01000$.

\subsubsection{Conclusions}

The Monte Carlo simulations validate the experimental results and demonstrate that the coherent normalization procedure for the $\mathrm{Cd}-109$ bone Gd x-ray fluorescence system is effective to better than 5\% (3.5\% for simulations, $2.5 \%$ for experimental) over a large range of tissue thicknesses (0-12.2 mm) for in vivo measurements. The variation in the 
experimentally measured $\mathrm{Gd}$ signal to coherent signal is $0.749 \pm 0.019$, and $0.717 \pm 0.025$

for the Monte Carlo simulation for a $120 \mu \mathrm{g} \mathrm{Gd} / \mathrm{g}$ plaster phantom. The normalization is able to correct for variation in overlying tissue thickness within the investigated range typical of an adult population, and will be helpful for in vivo Gd measurements to correct for interpatient variability.

\subsubsection{Acknowledgements}

The Natural Sciences and Engineering Research Council of Canada (FEM DRC), and the Ontario Graduate Scholarship (MLL) is acknowledged for financial support of this study. JLG would like to acknowledge the support of Ryerson University in the form of internal start-up grants.

\subsubsection{Conflict of interest}

The authors affirm that none of them has any conflict of interest. 


\section{Chapter 3: Conclusions and Future Work}

\subsection{Summary and Conclusions}

Gadolinium-based contrast agents (GBCA) used in magnetic resonance (MR) imaging have been in clinical use for the past three decades [4]. These agents are considered safe only if used in clinically approved doses and in patients with healthy renal function [9]. The safety of these agents has become an area of concern in the MR imaging community particularly after recent clinical and laboratory evidence of retained amounts of gadolinium in patients with normal kidney function who have been exposed [3] [9] [10]. In addition to the elevated levels of gadolinium in vivo, many individuals reported symptoms similar to those found in patients suffering a gadolinium related toxicity medical condition known as Nephrogenic Systemic Fibrosis (NSF). A group of medical professionals has proposed a term to label gadolinium toxicity in patients with normal kidney function; gadolinium deposition disease (GDD) [12].

Since detecting gadolinium in vivo is an important step in diagnosing any related medical conditions and potential long-term health effects [8], efforts were recently made to develop bone gadolinium measurement systems based on X-ray fluorescence (XRF), a non-invasive and painless analytical method [16]. Bone was selected due to evidence that the bone

matrix is the largest gadolinium deposition site [13]. The feasibility of a ${ }^{109} \mathrm{Cd}$ based XRF system to measure gadolinium in vivo has been confirmed [13][31], however, interpatient variability and other geometrical factors can affect the measurement making it dependent 
on patient body type and reducing the robustness of the measurement unless correction factors are applied.

The use of the coherent normalization as a correction procedure has been shown to produce measurements that are only a function of the amount of the element present in the volume being measured and independent of other factors such as the size and shape of the bone as well as OTT covering the bone at the measurement site [18]. In other words, the need for correction factors can be eliminated if the coherent normalization is feasible. For the coherent normalization to be effective as a correction method, four criteria must be fulfilled [18]. In the case of detecting gadolinium using the ${ }^{109} \mathrm{Cd}$ based XRF, two of the four criteria are not satisfied. Therefore, further investigation of the method was required.

Chapter 2 presents an investigation of the validity of the coherent normalization to correct for a wide range of overlying tissue thickness (OTT) of an adult population. The latest available release of Monte Carlo N-Particle (MCNP) radiation transport code, MCNP6.1.1 was used. This version was selected due to recent improvements which offer comprehensive modelling of the coherent peak compared to preceding ones. Figure A.1 (Appendix A) shows a simulated detector response.

Through the use of MCNP, we were able to provide a theoretical explanation of the feasibility of the coherent normalization method. The two fluence components, primary and secondary, contributing to the fluorescence of gadolinium were separated and studied as a function of OTT. Furthermore, we introduced and defined the Secondary Fluence Fluorescence Factor (SFFF). The SFFF provides an explanation for why the method is 
valid without satisfying all four criteria. The increase or build-up of secondary photons provides a secondary component to fluoresce $\mathrm{Gd}$ and counteracts the differential attenuation of the Gd K X-rays compared to the $88 \mathrm{keV}$ source photons. We concluded that experimental and simulated results are in agreement and that the coherent method is feasible and able to correct for the effect of varying OTT over a wide range $(0-12.2 \mathrm{~mm})$, representative of an adult population. 


\subsection{Future Work}

The ability of the coherent normalization method to correct for overlying tissue thickness (OTT) in a tibial X-ray fluorescence (XRF) gadolinium measurement system has been demonstrated through experimental work and Monte Carlo simulation. As an extension to this work, another crucial step to improve the performance of the current bone gadolinium XRF system is to investigate the validity of the coherent normalization to correct for the effect of varying bone size, another interpatient variability than can affect the measurement. A preliminary Monte Carlo based study was conducted in which a set of bone phantoms covering a range of bone radii $(0.75-2.5 \mathrm{~cm})$ were simulated. The initial results showed that the method has a potential of being able to make the measurement independent of bone size. A thorough investigation is still required for this correction.

Although the feasibility of the developed XRF system to detect gadolinium in bone has been confirmed, the minimum detectable limits of the system could be improved if a collimator of different material is used than the currently used tungsten collimator. As mentioned in Chapter 2, the gadolinium K X-ray peaks are located on the side of another feature of the collected spectra, which is the Compton backscattered tungsten $\mathrm{K} X$-rays from the collimator. Counts from this feature could be a reason for a higher than ideal signal-to-noise ratio in the gadolinium $\mathrm{K}_{\mathrm{a}} \mathrm{X}$-rays region. The use of a different material for the collimator is an area of investigation that can lead to enhancing system performance.

As mentioned earlier, the coherent conversion factor (CCF) is another useful factor to correct measurements for differences in coherent scattering between bone phantoms, as 
calibration standards, and the actual bone matrix. In another preliminary study using MCNP, the CCF was found to be 1.35 instead of the previously reported value of approximately 1.46 [29]. Since CCF is a vital correction for in vivo studies, this requires further detailed and careful examination.

The multiplication product of the normalized SFFF and the differential exponential attenuation plotted as function of OTT (Figure 2.4 in Chapter 2) shows a significant increase between 0 and $3.3 \mathrm{~mm}$ effecting the best fit line if the $0 \mathrm{~mm}$ thickness point was not included in the fitting which can result in a line of a relatively significant negative slope. Further investigation of this effect can be done using Monte Carlo simulations.

As mentioned in the article presented in Chapter 2, one limitation of this work is the use of Solid Water ${ }^{\circledR}$ to represent OTT. This may not be the best representation of the actual fat and skin layers found in a measurement site as photon interactions in the energy range of interest are largely dependent on material composition. This is an area of improvement where the use of better soft tissue equivalent material could be investigated.

The need and the use of a reliable XRF bone gadolinium measurement system are not limited to only detecting gadolinium in subjects who were exposed to MR imaging contrast agents. The presence of gadolinium has been detected in surface and drinking water [49]. As a result, it is also possible that gadolinium is making its way into the food chain through human consumed vegetation and animals [50]. Consequentially, a larger human population could be exposed to gadolinium through non-medical pathways. Since free gadolinium is highly toxic even in small amounts [50] and the potential long-term effects of gadolinium 
deposition are still unknown, a reliable XRF system can serve as a monitoring tool to detect trace amounts in exposed individuals. 


\section{Appendix A: MCNP Detector Spectrum}

Figure A.1 below shows a spectrum of the modelled XRF system. The code was written to tally pulse height in one detector (Det02). The Gaussian energy broadening (GEB) card was not included for this simulation. The $\mathrm{Gd} \mathrm{K}_{\alpha} \mathrm{X}$-ray intensities (or counts) where determined by subtracting a linear background. The coherent ratio was determined by taking the ratio of the $\mathrm{Gd} \mathrm{K}_{\alpha} \mathrm{X}$-ray intensity to the coherent peak intensity.

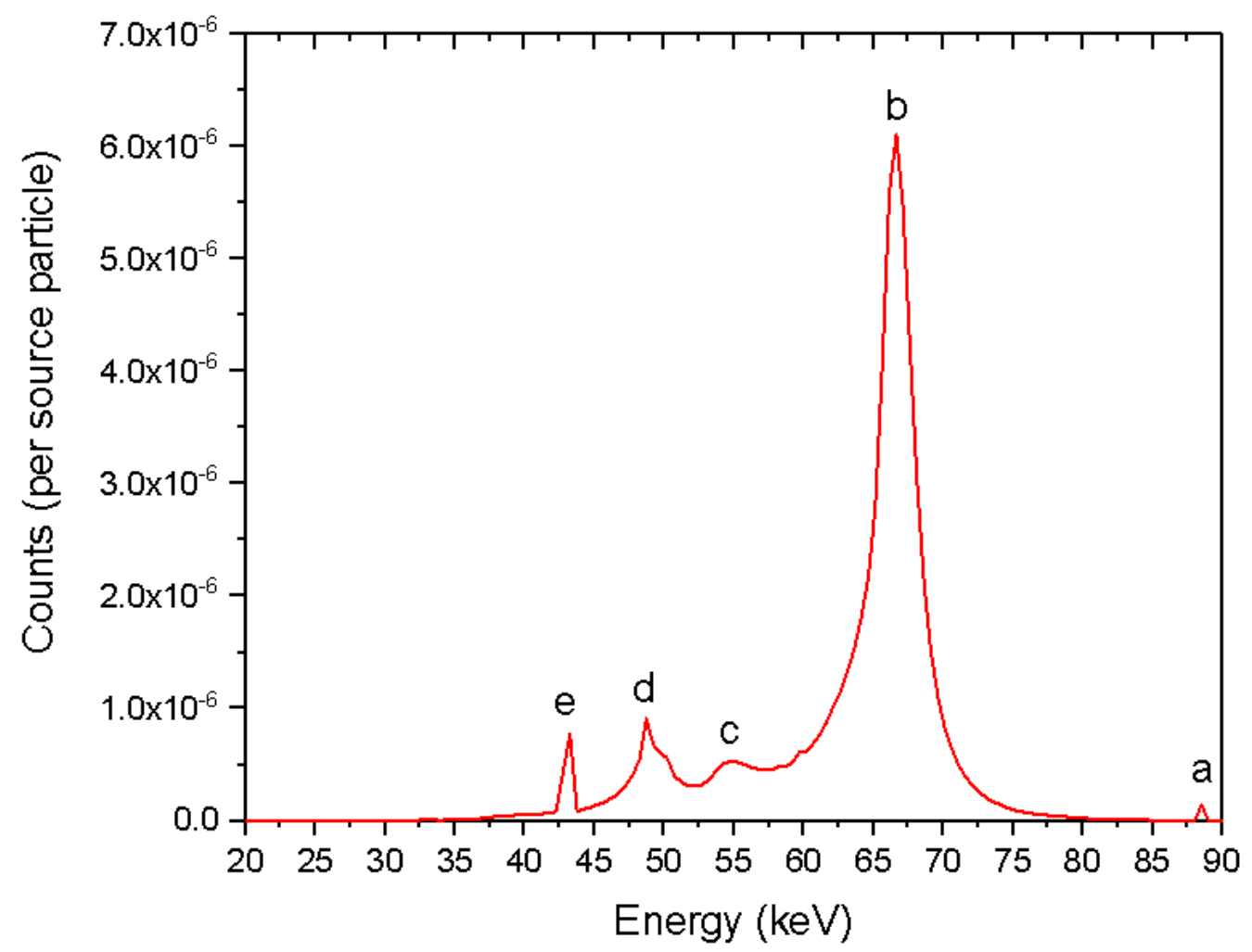

Figure A.1: MCNP detector spectrum. a) Coherent peak, b) Compton peak, c) Compton scattered photons that penetrate the detection volume and scatter back into the detector, d) Backscattered tungsten $\mathrm{K}_{\mathrm{a}} \mathrm{X}$-rays from the collimator material and gadolinium $\mathrm{K}_{\beta} \mathrm{X}$-rays, e) Gadolinium $\mathrm{K}_{\alpha}$ X-rays. 


\section{References}

[1] P. Caravan, J. Ellison, T. McMurry and R. Lauffer, "Gadolinium (III) Chelates as MRI Contrast Agents: Structure, Dynamics, and Applications", Chemical Reviews, vol. 99, no. 9, pp. 2293-2352, 1999.

[2] S. Aime, L. Frullano and S. Geninatti Crich, "Compartmentalization of a Gadolinium Complex in the Apoferritin Cavity: A Route To Obtain High Relaxivity Contrast Agents for Magnetic Resonance Imaging", Angewandte Chemie, vol. 114, no. 6, pp. 1059-1061, 2002.

[3] A. Malayeri, K. Brooks, L. Bryant, R. Evers, P. Kumar, D. Reich and D. Bluemke, "National Institutes of Health Perspective on Reports of Gadolinium Deposition in the Brain", Journal of the American College of Radiology, vol. 13, no. 3, pp. 237-241, 2016.

[4] M. Rogosnitzky and S. Branch, "Gadolinium-based contrast agent toxicity: a review of known and proposed mechanisms", BioMetals, vol. 29, no. 3, pp. 365-376, 2016.

[5] S. Aime and P. Caravan, "Biodistribution of gadolinium-based contrast agents, including gadolinium deposition", Journal of Magnetic Resonance Imaging, vol. 30, no. 6, pp. 1259-1267, 2009.

[6] P. Ngoya, Z. Vawda and J. Lotz, "Nephrogenic systemic fibrosis (NSF) and gadolinium-based contrast media", South African Journal of Radiology, vol. 17, no. 3, 2013.

[7] K. Kitajima, T. Maeda, S. Watanabe, Y. Ueno and K. Sugimura, "Recent topics related to nephrogenic systemic fibrosis associated with gadolinium-based contrast agents", International Journal of Urology, vol. 19, no. 9, pp. 806-811, 2012.

[8] R. Semelka, M. Ramalho, M. AlObaidy and J. Ramalho, "Gadolinium in Humans: A Family of Disorders", American Journal of Roentgenology, vol. 207, no. 2, pp. 229-233, 2016.

[9] N. Murata, L. Gonzalez-Cuyar, K. Murata, C. Fligner, R. Dills, D. Hippe and K. Maravilla, "Macrocyclic and Other Non-Group 1 Gadolinium Contrast Agents Deposit Low Levels of Gadolinium in Brain and Bone Tissue", Investigative Radiology, vol. 51, no. 7, pp. 447-453, 2016. 
[10] T. Kanda, T. Fukusato, M. Matsuda, K. Toyoda, H. Oba, J. Kotoku, T. Haruyama, K. Kitajima and S. Furui, "Gadolinium-based Contrast Agent Accumulates in the Brain Even in Subjects without Severe Renal Dysfunction: Evaluation of Autopsy Brain Specimens with Inductively Coupled Plasma Mass Spectroscopy", Radiology, vol. 276, no. 1, pp. 228$232,2015$.

[11] J. Ramalho, M. Ramalho, M. Jay, L. Burke and R. Semelka, "Gadolinium toxicity and treatment", Magnetic Resonance Imaging, vol. 34, no. 10, pp. 1394-1398, 2016.

[12] R. Semelka, J. Ramalho, A. Vakharia, M. AlObaidy, L. Burke, M. Jay and M. Ramalho, "Gadolinium deposition disease: Initial description of a disease that has been around for a while", Magnetic Resonance Imaging, vol. 34, no. 10, pp. 1383-1390, 2016.

[13] M. Lord, F. McNeill, J. Gräfe, M. Noseworthy and D. Chettle, "A phantom-based feasibility study for detection of gadolinium in bone in-vivo using X-ray fluorescence", Applied Radiation and Isotopes, vol. 112, pp. 103-109, 2016.

[14] F. Mostafaei and L. Nie, "The study of in vivo X-ray fluorescence (XRF) technique for gadolinium (Gd) measurements in human bone", Journal of Instrumentation, vol. 11, no. 08, pp. T08001-T08001, 2016.

[15] B. Beckhoff, B. Kanngießer, N. Langhoff, R. Wedell and H. Wolff, Handbook of Practical X-Ray Fluorescence Analysis. Berlin, Heidelberg: Springer-Verlag Berlin Heidelberg, 2006.

[16] D. Chettle, "In vivo applications of X-ray fluorescence in human subjects", Pramana, vol. 76, no. 2, pp. 249-259, 2011.

[17] K. Imamura, Y. Sasaki, N. Sekita, K. Someya and M. Fuji, "Quantitative In Vivo Measurement of Thyroidal Iodine Content by Static X-Ray Fluorescent Technique: Some Preliminary Clinical Observations", Investigative Radiology, vol. 14, no. 4, pp. 316-322, 1979.

[18] L. Somervaille, D. Chettle and M. Scott, "In vivo measurement of lead in bone using X-ray fluorescence", Physics in Medicine and Biology, vol. 30, no. 9, pp. 929-943, 1985.

[19]L. Ahlgren and S. Mattsson, "Cadmium in man measured in vivo by X-ray fluorescence analysis", Physics in Medicine and Biology, vol. 26, no. 1, pp. 19-26, 1981.

[20]A. Pejović-Milić, I. Stronach, J. Gyorffy, C. Webber and D. Chettle, "Quantification of bone strontium levels in humans by in vivo x-ray fluorescence", Medical Physics, vol. 31, no. 3, pp. 528-538, 2004. 
[21] J. O'Meara, D. Chettle, F. McNeill and C. Webber, "In vivo X-ray fluorescence (XRF) measurement of uranium in bone", Applied Radiation and Isotopes, vol. 49, no. 5-6, pp. 713-715, 1998.

[22] J. Nguyen, Z. Keldani, E. Da Silva, A. Pejović-Milić and J. Gräfe, "The feasibility of in vivo detection of lanthanum using a 241 Am K x-ray fluorescence system", Physiological Measurement, vol. 38, no. 9, pp. 1766-1775, 2017.

[23] E. Podgoršak, Radiation physics for medical physicists. Heidelberg: Springer, 2010.

[24] F. E. McNeill and J. M. O'Meara, "The In Viva Measurement of Trace Heavy Metals by K X-Ray Fluorescence," International Centre for Diffraction Data (ICDD), Denver, 1999.

[25] M. Zamburlini, A. Pejović-Milić and D. Chettle, "Coherent normalization of finger strontium XRF measurements: feasibility and limitations", Physics in Medicine and Biology, vol. 53, no. 15, pp. N307-N313, 2008.

[26] J. M. O'Meara, D. R. Chettle and F. E. McNeill, "The Validity of the Coherent Scatter Peak Normalization of X-ray Intensities Detected During in vivo XRF," International Centre for Diffraction Data (ICDD), 1999.

[27] J. O'Meara and D. Fleming, "Uncertainty calculations for the measurement of in vivo bone lead by x-ray fluorescence", Physics in Medicine and Biology, vol. 54, no. 8, pp. 2449-2461, 2009.

[28] A. Todd, M. Arnold, A. Aro, D. Chettle, D. Fleming, F. McNeill, E. Moshier, H. Nie and I. Stronach, "Corrections to "How to calculate lead concentration and concentration uncertainty in XRF in vivo bone lead analysis" by Kondrashov and Rothenberg", Applied Radiation and Isotopes, vol. 58, no. 1, pp. 41-50, 2003.

[29] A. Todd, "Coherent scattering and matrix correction in bone-lead measurements", Physics in Medicine and Biology, vol. 45, no. 7, pp. 1953-1963, 2000.

[30] E. Da Silva, B. Kirkham, D. Heyd and A. Pejović-Milić, "Pure Hydroxyapatite Phantoms for the Calibration of in Vivo X-ray Fluorescence Systems of Bone Lead and Strontium Quantification", Analytical Chemistry, vol. 85, no. 19, pp. 9189-9195, 2013.

[31] M. Lord, F. McNeill, J. Gräfe, A. Galusha, P. Parsons, M. Noseworthy, L. Howard and $\mathrm{D}$. Chettle, "Confirming improved detection of gadolinium in bone using in vivo XRF", Applied Radiation and Isotopes, vol. 120, pp. 111-118, 2017. 
[32] T. Kanda, K. Ishii, H. Kawaguchi, K. Kitajima and D. Takenaka, "High Signal Intensity in the Dentate Nucleus and Globus Pallidus on Unenhanced T1-weighted MR Images: Relationship with Increasing Cumulative Dose of a Gadolinium-based Contrast Material", Radiology, vol. 270, no. 3, pp. 834-841, 2013.

[33] Y. Errante, V. Cirimele, C. Mallio, V. Di Lazzaro, B. Zobel and C. Quattrocchi, "Progressive Increase of T1 Signal Intensity of the Dentate Nucleus on Unenhanced Magnetic Resonance Images Is Associated With Cumulative Doses of Intravenously Administered Gadodiamide in Patients With Normal Renal Function, Suggesting Dechelation", Investigative Radiology, vol. 49, no. 10, pp. 685-690, 2014.

[34] R. McDonald, J. McDonald, D. Kallmes, M. Jentoft, D. Murray, K. Thielen, E. Williamson and L. Eckel, "Intracranial Gadolinium Deposition after Contrast-enhanced MR Imaging", Radiology, vol. 275, no. 3, pp. 772-782, 2015.

[35] C. Quattrocchi, C. Mallio, Y. Errante, V. Cirimele, L. Carideo, A. Ax and B. Zobel, "Gadodiamide and Dentate Nucleus T1 Hyperintensity in Patients With Meningioma Evaluated by Multiple Follow-Up Contrast-Enhanced Magnetic Resonance Examinations With No Systemic Interval Therapy", Investigative Radiology, vol. 50, no. 7, pp. 470-472, 2015.

[36] J. Ramalho, M. Ramalho and R. Semelka, "Gadolinium Deposition and Toxicity: A Global Concern", Current Radiology Reports, vol. 4, no. 11, 2016.

[37] W. Gibby, K. Gibby and W. Gibby, "Comparison of Gd DTPA-BMA (Omniscan) versus Gd HP-DO3A (ProHance) Retention in Human Bone Tissue by Inductively Coupled Plasma Atomic Emission Spectroscopy", Investigative Radiology, vol. 39, no. 3, pp. 138142, 2004.

[38] G. White, W. Gibby and M. Tweedle, "Comparison of Gd(DTPA-BMA) (Omniscan) Versus Gd(HP-DO3A) (ProHance) Relative to Gadolinium Retention in Human Bone Tissue by Inductively Coupled Plasma Mass Spectroscopy", Investigative Radiology, vol. 41, no. 3, pp. 272-278, 2006.

[39] T. Darrah, J. Prutsman-Pfeiffer, R. Poreda, M. Ellen Campbell, P. Hauschka and R. Hannigan, "Incorporation of excess gadolinium into human bone from medical contrast agents", Metallomics, vol. 1, no. 6, p. 479-488, 2009.

[40] A. Sherry, P. Caravan and R. Lenkinski, "Primer on gadolinium chemistry", Journal of Magnetic Resonance Imaging, vol. 30, no. 6, pp. 1240-1248, 2009. 
[41] J. O'Meara, D. Chettle, F. McNeill and C. Webber, "The feasibility of measuring bone uranium concentrationsin vivousing source excited K x-ray fluorescence", Physics in Medicine and Biology, vol. 42, no. 6, pp. 1109-1120, 1997.

[42] J. O’Meara, J. Börjesson, D. Chettle and S. Mattsson, "Normalisation with coherent scatter signal: improvements in the calibration procedure of the -based in vivo XRF bone$\mathrm{Pb}$ measurement", Applied Radiation and Isotopes, vol. 54, no. 2, pp. 319-325, 2001.

[43] T Goorley, "MCNP6 1.1-beta release notes", Los Alamos National Laboratory, 2014.

[44] T. Goorley, M. James, T. Booth, F. Brown, J. Bull, L. Cox, J. Durkee, J. Elson, M. Fensin, R. Forster, J. Hendricks, H. Hughes, R. Johns, B. Kiedrowski, R. Martz, S. Mashnik, G. McKinney, D. Pelowitz, R. Prael, J. Sweezy, L. Waters, T. Wilcox and T. Zukaitis, "Initial MCNP6 Release Overview", Nuclear Technology, vol. 180, no. 3, pp. 298-315, 2012.

[45] G. Hughes, "Recent developments in low-energy electron/photon transport for MCNP6", Progress in Nuclear Science and Technology, vol. 4, pp. 454-458, 2014.

[46] G. Hughes, "Enhanced electron-photon transport in MCNP6", in 2013-Joint International Conference on Supercomputing in Nuclear Applications + Monte Carlo, 2014, p. 3105.

[47] E. Podgorsak, Radiation physics for medical physicists. Springer, 2006.

[48] A. Pejović-Milić, J. Brito, J. Gyorffy and D. Chettle, "Ultrasound measurements of overlying soft tissue thickness at four skeletal sites suitable forin vivox-ray fluorescence", Medical Physics, vol. 29, no. 11, pp. 2687-2691, 2002.

[49] M. Birka, C. Wehe, O. Hachmöller, M. Sperling and U. Karst, "Tracing gadoliniumbased contrast agents from surface water to drinking water by means of speciation analysis", Journal of Chromatography A, vol. 1440, pp. 105-111, 2016.

[50] H. Thomsen, "Are the increasing amounts of gadolinium in surface and tap water dangerous?", Acta Radiologica, vol. 58, no. 3, pp. 259-263, 2016. 